\title{
Influence of Different Textures on Machining Performance of a Milling Tool
}

\author{
Jianfeng Pan $\left(\mathbb{D},{ }^{1}\right.$ Kai Feng $\left(\mathbb{D},{ }^{2}\right.$ Lihua He $\mathbb{D}^{2},{ }^{2}$ Kai Zhuang $\mathbb{D}^{2},{ }^{2}$ Jing Ni $\mathbb{D}^{2},{ }^{2}$ and Junjie Lv $\mathbb{D}^{2}$ \\ ${ }^{1}$ Special Equipment Institute, Hangzhou Vocational and Technical College, Hangzhou, China \\ ${ }^{2}$ School of Mechanical Engineering, Hangzhou Dianzi University, Hangzhou 310005, China \\ Correspondence should be addressed to Lihua He; helihua0617@yahoo.com
}

Received 17 August 2020; Revised 27 October 2020; Accepted 15 November 2020; Published 25 November 2020

Academic Editor: Victor M. Castaño

Copyright (c) 2020 Jianfeng Pan et al. This is an open access article distributed under the Creative Commons Attribution License, which permits unrestricted use, distribution, and reproduction in any medium, provided the original work is properly cited.

Milling plays a significant role in the machining of aluminum alloy parts. However, in the milling process, the material easily adheres to the tip of the milling tool due to the high ductility and thermal deformation of the aluminum alloy; the machining efficiency and quality are hard to further improve. Hence, in order to promote the machining performances, three textures (linear, wavy, and micropitted) are prepared, respectively, on rake face of milling tools using the laser processing system. Then, milling load, top-burrs, and surface quality are chosen to discuss the machining performance of textured milling tools. In particular, the top-burrs are investigated by relative area ratio of top-burrs (RARB) which is calculated from the micrograph after binarization. The obtained results evidently show that the wavy textured milling tool reduces the milling load by an amount of $10.7 \%$ compared to the nontextured milling tool. This is due to the smaller contact area that reduces the internal friction of the tool-chip contact area and thus reduces the load. And surface roughness improves by an amount of $23.8 \%$ because the wavy texture has the largest proportion of the unit area, and it can effectively improve the storage capacity of debris. Therefore, the wavy texture proposed in this research is of great reference value for the optimization of the machining performance of the milling tool.

\section{Introduction}

Milling occupies a significant position in mechanical processing because of its high efficiency and high accuracy. Also, the performance of the milling tool has a great impact on the surface roughness and quality of the workpiece $[1,2]$. However, in the cutting process of aluminum alloy parts, the chips are severely adhered to the surface of milling tools owing to high temperature generated by the friction at the tool-chip contact interfaces and low melting point and high ductility of the material [3]. Thus, the machining efficiency and quality of the milling tool are reduced due to the adhesion of the material on the tool [4]. In this regard, there is a need to implement the new technique in milling operation which can provide considerable reduction in adhesion occurring at the contact interfaces. The surface texturing technology is introduced to cutting tools with an aim to minimize the adhesion caused by friction at the cutting regime during metal removal processes [5]. Moreover, although a blossom of interest has been exhibited in the past ten years in the field of textured tools which includes their multifarious applications such as the turning tool and disk milling tool, the application report of texture on the milling tool is scarce. Therefore, it is very necessary to research an economical and efficient texture for the milling tool [6-9].

In recent years, several studies have been performed toward the effect of textures in cutting tools. Enomoto and Tatsuya processed the groove texture in micro and nanoscale on the rake face of a carbide tool. By evaluating the size parameter of various textures, they concluded that application of textures decreased the friction, and on the 
condition of the groove texture with a large width, chips easily enter and form blockages [10].

Koshy and Tovey used electrical discharge machining (EDM) to process textures on the surface of the turning tool. They reported that the existence of the texture promoted the improvement of the friction state of the tool-chip interface and brought about a $30 \%$ and $13 \%$ reduction in the average feed and cutting forces, respectively [11].

Toshiyuki et al. examined the effectiveness of four different microtextures at the rake face on the friction force and the coefficient of friction in machining aluminum alloy A6061-T6. The results revealed that parallel and dot-type microtextures reduced more effectively the friction force and the coefficient of friction. It was also found that the microtexture became more effective as the pattern size of microtexture decreased or the depth of texture increased [12].

Tiffany Davis et al. applied rectangular surface textures on the margins of drill bits, and the textured drills were tested by drilling a series of holes in a titanium plate. The obtained results indicated good performance of textures in reduction of adhesion of titanium chips on the drill margins, improving the lifetime of drill bits [13].

Xie et al. evaluated the effect of $V$-shaped microgrooves in terms of cutting temperature, cutting force, and tool wear on the turning tool. The achieved results showed that application of this texture reduced the chip friction and eliminated cutting heat [14].

Noritaka et al. studied the machining performance of a textured diamond tool during cutting of aluminum alloy and nickel phosphorus. The results indicated the textured tool showed excellent decrease in friction at the tool-chip interface. In addition, a set of experiments showed that the magnitude of the decrease effect depends on the shape and direction of the texture [15].

And due to precise dimensional control and generated feature quality, the laser beam micromachining technique qualified to be the best technique for creating microtextures by Naveed et al. [16].

Anis et al. used the femtosecond laser system in preparing groove texture on the surface of cemented carbide tools. They reported that the texture alleviate the critical contact condition of the tool-chip flow surface, thereby reducing the cutting force and feed force [17].

Liu et al. applied different kinds of textures on the flank faces of WC/Co carbide tools by laser. And dry cutting on green alumina ceramic was carried out with conventional cemented carbide and textured cutting tools. The obtained results indicated good performance of grooves parallel to the main cutting edge in improvement of wear resistance [18].

Vasumathy and Meena studied the effect of different lay directions of the textures with respect to the chip flow. According to their results, the cutting force and friction coefficient was reduced due to the textures modified in the adhesion of chips on the rake face compared to the conventional tool [19].

According to the abovementioned literature, most of the researchers have investigated the effect of textures in various tools such as the turning tool, grinding tool, and drill tool. Very few studies have been carried out toward the application of textures in the curved surface of rake face of the milling tool; therefore, three types of textures were prepared on the milling tool with the laser processing system. Then, the comparative experiments were carried out on a CNC milling machine. And in these studies, the effect of textures have been evaluated on the milling performance such as milling load, top-burrs, and surface roughness. To the best of authors' knowledge, there have been few works concerning the effect of various textured milling tools on residual burrs. Hence, the main goal of the present study is to investigate the various textures effect on surface quality during the milling process of aluminum alloy by means of the image processing technique. Furthermore, it is for the first time that the RARB is used as the evaluation methodology to evaluate the amount of top-burrs. Top-burrs were concerned in this work due to their impacts on the cost of postprocessing and assembly precision of the workpiece.

\section{Experiment}

2.1. Experiment System. The experimental tests were carried out on aluminum alloy using different milling tools with a diameter of $8 \mathrm{~mm}$ and performed in dry milling. The aluminum alloy 6061 with the dimension of $40 \mathrm{~mm} \times 30 \mathrm{~mm} \times 10 \mathrm{~mm}$ was chosen as the workpiece. As shown in Figure 1, the milling machine was the vertical machining center (type: FEELER VMP-23A), and the processing parameters are given in Table 1 . The milling load was acquired by means of a piezoelectric sensor (type: KISTLER 9119AA2) with its acquisition frequency of $1024 \mathrm{~Hz}$. It can be seen in Figure 1, that the milling tool rotated clockwise and was fed in the negative direction of $y$.

The milling tests were performed in four different conditions, namely, with three textured milling tools and with nontextured milling tools. In this experiment, the textures were prepared on the rake face of the milling tools by a laser-marking machine (Han's Laser H20) as shown in Figure 1, and the parameters of the laser system are also given in Table 1. The tungsten-steel end milling tools with three blades were chosen as the test object. Since it was difficult to fabricate the texture on a curved surface, the milling tool was fixed on a vise holder with an angleadjusting function. Three types of textures such as linear groove, wavy groove, and micropit with a depth of $50 \mu \mathrm{m}$ were prepared, respectively, on the rake face of tools. As given in Figure 1 and Table 2, the linear texture and the wavy texture were continuous grooves with a line width of $d$, and the distance between each groove was $b$. The micropitted textures were distributed in an array on the rake face of milling tools, and the pitch dimension is $b$, and also, the diameter of the pattern is $d$. Specially, the surface was treated with 2000 mesh sandpaper preliminarily and 5000 mesh sandpaper subsequently as the laser processing was completed. Finally, the processed milling tools were placed in an ultrasonic mixer for 30 minutes to remove impurities. 


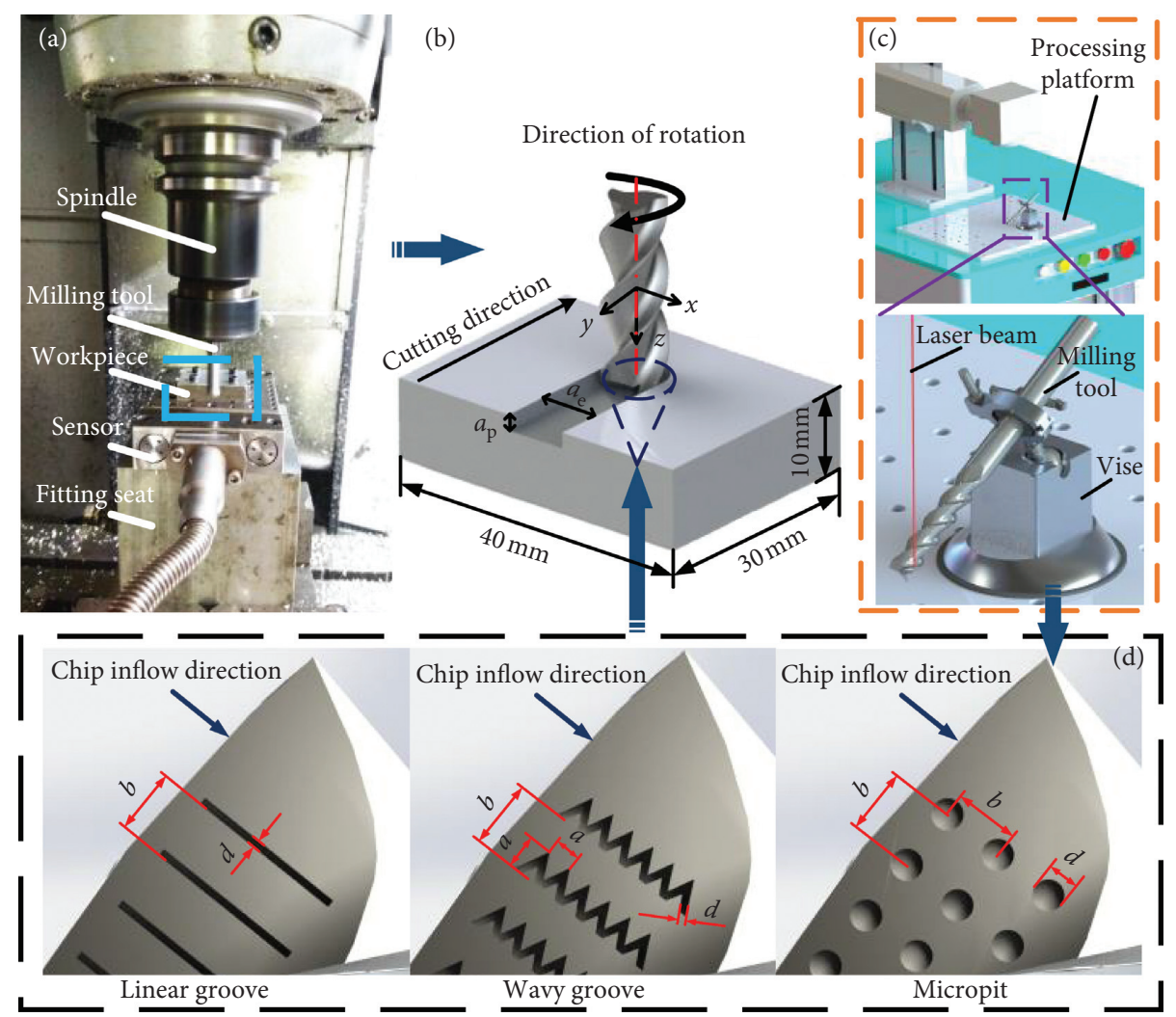

FIGURE 1: Milling test system of textured tools. (a) Milling experiment; (b) cutting diagram of the milling cutter; (c) the textures are prepared on the milling tools; (d) schematic diagram of textures at the tip.

TABLE 1: Parameters of the experiment system.

\begin{tabular}{lccc}
\hline \multicolumn{2}{l}{ Parameters of the laser system } & \multicolumn{2}{c}{ Parameters of the milling test } \\
Parameter & Value & Parameters & Value \\
\hline Power & $12 \mathrm{~W}$ & Spindle speed $(\mathrm{r} / \mathrm{min})$ & 3000 \\
Spot diameter & $50 \mu \mathrm{m}$ & Feed speed $\nu_{f}(\mathrm{~mm} / \mathrm{min})$ & 200 \\
Frequency & $60 \mathrm{kHz}$ & Milling depth $a_{p}(\mathrm{~mm})$ & 2 \\
Processing times & 20 & Milling width $a_{e}(\mathrm{~mm})$ & 8 \\
Repeat accuracy & $\pm 0.003 \mathrm{~mm}$ & Cooling method & Dry \\
\hline
\end{tabular}

\section{Experimental Evaluation}

3.1. Milling Load. During metal cutting, the cutting load is a critical physical parameter, which directly affects cutting heat, tool wear, and durability. And also, it has an impact on the machining accuracy of the workpiece and the quality of the machined surface [20]. At the same time, in the process of programming the cutting conditions, the cutting load is the necessary basis for computing the power of the bed, determining the cutting parameters, monitoring the cutting status, and designing the tool holder. In this research, three component loads, $F_{x}, F_{y}$, and $F_{z}$, are acquired, and the resultant load $F_{n}$ is used to evaluate the milling load of different textured tools. Among them, the resultant load is expressed by the following formula:

$$
F_{n}=\sqrt{\left(F_{x}^{2}+F_{y}^{2}+F_{z}^{2}\right)}
$$

3.2. Top-Burr. Burrs are unavoidable residual products caused by plastic deformation during the cutting process, and their existence will inevitably bring a lot of subsequent problems, such as the finishing, assembly process, transportation, and later use. According to the research and classification of burrs by Gillespie in the process of milling through grooves, the burr distributes along the edges where the top and transition faces intersect and are called top-burr [21]. Therefore, in this paper, the top-burrs of the workpiece are focused as the top-burrs are generally large in size $[22,23]$.

It is observed that the burrs located in the cut-in, cut-out, and top areas are relatively large after cutting. To avoid the error caused by the uneven bending of the top-burrs, two plates were used to flatten them along the edges, as shown in Figure 2. The AISI 1045 steel with a mass of $320 \mathrm{~g}$ and a size of $40 \mathrm{~mm} \times 20 \mathrm{~mm} \times 5 \mathrm{~mm}$ was selected as the plate. First, the plates were placed on the processed surface at an angle of $90^{\circ}$ and perpendicular to the unprocessed surface. Then, the two plates were slowly rotated to both sides with the rotation point (that is, the edge of the top of the groove) as the center, when the plates were in level with the unprocessed surface, and the plates were held as the briquettings on top of the workpiece.

After the workpiece and the briquetting stood for 24 hours, observe the top-burr. A high-speed digital camera (type: KEYENCE VW-9000) was used to photograph the middle area of the top-burrs, as shown in Figure 2. 
TABLE 2: Parameter of textured size.

\begin{tabular}{lccc}
\hline Type & $b(\mathrm{~mm})$ & $d(\mathrm{~mm})$ & $a(\mathrm{~mm})$ \\
\hline Linear groove & 0.4 & 0.05 & - \\
Wavy groove & 0.4 & 0.05 & 0.2 \\
Micropit & 0.4 & 0.2 & - \\
\hline
\end{tabular}

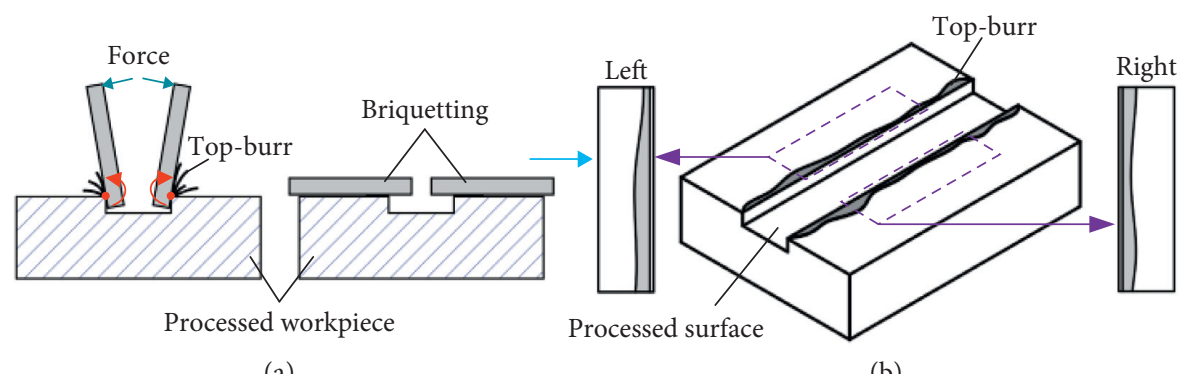

(a)

(b)

FIGURE 2: Schematic diagram of the measuring method of top-burr. (a) Top-burr flattening diagram; (b) schematic diagram of the sampling area.

3.3. Surface Quality. In general, surface morphology can qualitatively reflect the quality of the surface of the workpiece, and surface roughness can quantitatively evaluate the surface quality of the workpiece [24]. That is, the two can complement each other. Surface roughness is caused by the geometry of the tool and the feed movement during the cutting process. The parts with large surface roughness have low contact stiffness and poor abrasion resistance and are easy to wear. Therefore, in this paper, the arithmetic average surface roughness (Ra) and profile unit average width (RSm) are applied to evaluate the influence of textured tools on the surface quality.

\section{Results and Discussion}

4.1. Milling Load. The different textured tools are used to mill the workpiece under the same working conditions, and each textured tool is subjected to five milling tests. Then, a set of data with the maximum load is deleted and so is that with the minimum load. Finally, the remaining three sets of consistent data are selected to analyze. The change of milling load component $\left(F_{x}, F_{y}\right.$, and $\left.F_{z}\right)$ in three directions are shown in Figure 3.

In this experiment, the data of the stable cutting region (2-9s) is choosed to evaluate the milling performance because there is a relatively large vibration when the tool cuts in and out of the workpiece. In the stable region, $F_{x}$ is positive, and $F_{y}$ and $F_{z}$ are negative due to the milling direction. In order to intuitively reflect the trend of the milling load, the absolute values of $F_{y}$ and $F_{z}$ are selected to obtain their average values. Finally, the overall change in the milling load of different textured tools is evaluated by the resultant load $F_{n}$, as shown in Figure 4.

According to the analysis of resultant load $F_{n}$ of milling, the nontextured milling tool has the maximum resultant load $F_{n}$ of $98.5 \mathrm{~N}$, and the standard deviation is $1.09 \mathrm{~N}$.
Compared with the nontextured milling tool, the wavy textured milling tool has the minimum resultant load $F_{n}$ of $88 \mathrm{~N}$, which is reduced by $10.7 \%$, and the standard deviation is $0.88 \mathrm{~N}$. The resultant load $F_{n}$ of the linear textured milling tool is $92.4 \mathrm{~N}$, which is reduced by $6.2 \%$, and the standard deviation is $3.87 \mathrm{~N}$. For the micropit textured milling tool, the resultant load $F_{n}$ is reduced by $9.1 \%$, and its value was $89.5 \mathrm{~N}$, and the standard deviation is $3.47 \mathrm{~N}$. Whether the upper limit or the lower limit of the standard deviation is taken, the average load trend in each condition is still consistent. To sum up, in terms of reducing the load, the wavy textured milling tool had the best load-reducing performance, followed by the micropit milling tool and the linear textured milling tool. The experimental results showed that the milling tools with textures had a lower milling load than those without textures.

As shown in Figure 5(a), when the tool cut the plastic metal, high temperature and high pressure were generated between the chip and the rake face, so that the bottom layer of the chip was softened and stuck to the rake face near the tip [25], forming a bonding area of length $l_{1}$. The relative shear slip between the bonding layer and the upper metal layer belonged to internal friction, and its unit tangential force was equal to the shear yield limit of the material. Before the chip left the rake face, it only contacted at some protruding points. That is, in the sliding area with a length of $l_{2}$, the friction between the chip and the rake face was external friction at this moment. According to Yan et al. [26], internal friction was much larger than external friction because of the material's flow stress characteristics and the size of the bonding area. Accordingly, the texture fabricated on the tool surface can reduce the contact area between the chip and the rake surface. As shown in Figure 5(b), the contact area between the bonding layer and the rake surface was reduced. Thereby, the bonding layer was easily detached from the rake surface during the shear slippage, reducing internal friction. 


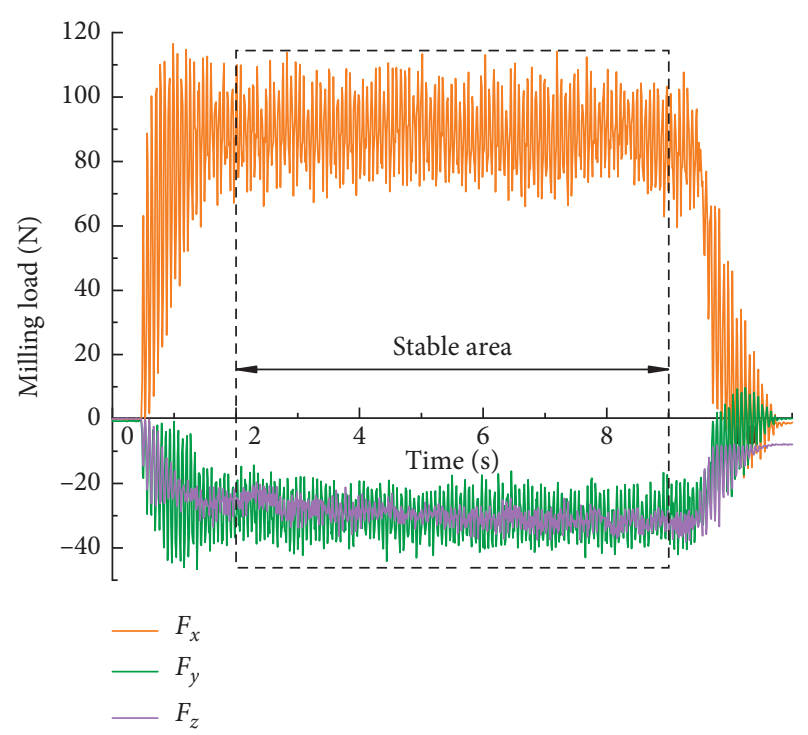

FIGURE 3: Signal images of the milling load component in three directions.

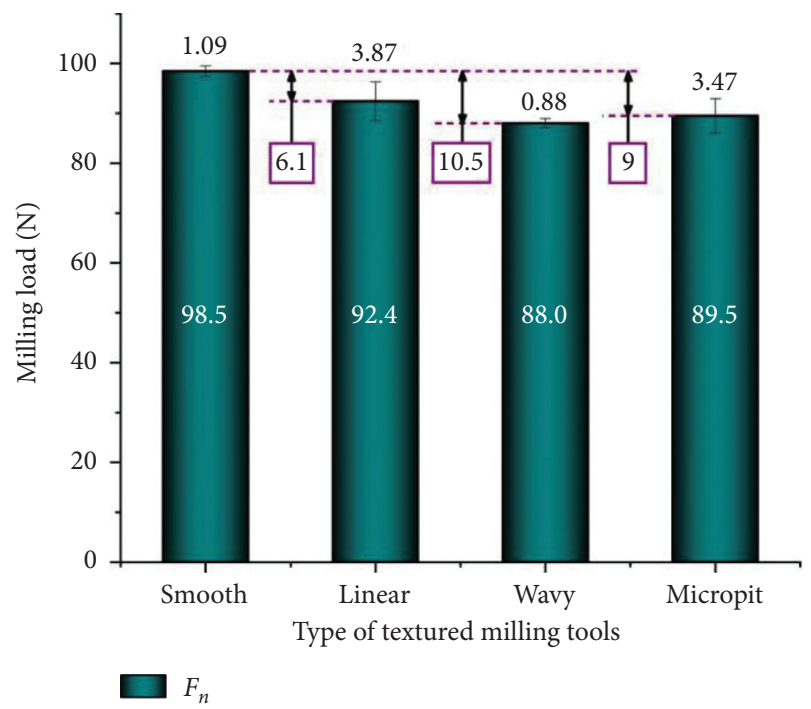

FIgURE 4: The resultant load of milling from different textured tools.

And at the sliding area, the external friction force was correspondingly reduced since the reduction of the tool-chip contact area.

To further investigate the effect of different textures on the milling load, an area of $1 \mathrm{~mm} \times 1 \mathrm{~mm}$ was selected on the textured tool, and then, the proportion of different textured areas per unit area was calculated according to the design size of the texture, as shown in Figure 6. The unit area ratio of wavy texture accounted for a maximum of $31.9 \%$, micropitted texture accounted for $28.3 \%$, and linear texture had the smallest unit area ratio accounting for $15 \%$. On the basis of the principle described in Figure 5, there was the minimal internal friction because the smaller contact area made the adhesive layer easily disengage at the tip bonding area $l_{1}$; the same may happen at the sliding area $l_{2}$. Therefore, the lowest milling load achieved during the cutting process with the wavy textured tool as the contact area between the chip and the tool surface was the smallest.

4.2. Top-Burr. The main reason for the formation of topburrs is that the material lacks support when cutting the edges of the top. Under the action of the cutting force, the workpiece material slips outwards, causing plastic deformation. These materials that have not been effectively removed will not form chips and accumulate in the form of burrs on the edge of the processed surface [27]. The number of burrs on the top of workpieces machined by different textured milling tools are significantly diverse. Some of them are folded toward the nonmachined surface, and some are even bonded to the nonprocessed surface. Seyed Ali and Victor [28] used the width of the burr on the top surface to evaluate the number of burrs on the workpiece.

As shown in Figure 7, the photomicrographs of the top of the workpiece were taken at a magnification of 50 times and with a pixel size of $426 \times 1417$. The burrs are randomly distributed in black on both sides of the milling groove. Although the burrs are connected in pieces, they are clearly distinguished from the color of the workpiece. So, digital image processing technology is applied to evaluate the number of top-burrs in this research. In order to accurately analyze the differences among them, the actual photomicrographs are binarized, and during this course, the isolated points in the unprocessed area are corroded to eliminate interference factors. Whereafter, the total number of black and white pixels in the whole binarized photograph is counted, and the same applies to the number of white pixels. Then, the proportion of burrs can be expressed as the ratio of the numbers in white to the total pixels for evaluation of the quality of the edges of the workpiece after milling. In this experiment, the cutting directions of the milling tool on the left and right sides of the through groove are different; so, the number of burrs on the both sides is discussed separately.

The average proportion of burrs can be taken from the manipulation of the photomicrographs of the five workpieces, as shown in the statistical chart of Figure 8 . There is the minimum proportion of burrs produced by the wavy textured tool whatever on the left or right sides, being $10.7 \%$ and $14.8 \%$, respectively. The top-burrs account for the maximum proportion of $31.6 \%$ and $36.7 \%$ under the milling condition of a nontextured tool. There are more burrs on the right than that on the left because the left side is upmilling and the right side is downmilling. This is because during downmilling, the material of the workpiece is extruded and sheared by the tool and moves from inside to the boundary, and the support stiffness at the boundary is small, and this part of the chip cannot be separated from the body material and eventually remains on the side of the boundary, forming fine burrs; in upmilling, the material that is squeezed and sheared is easily formed by the support of the body material; so, fewer burrs are generated, and the size is smaller. 


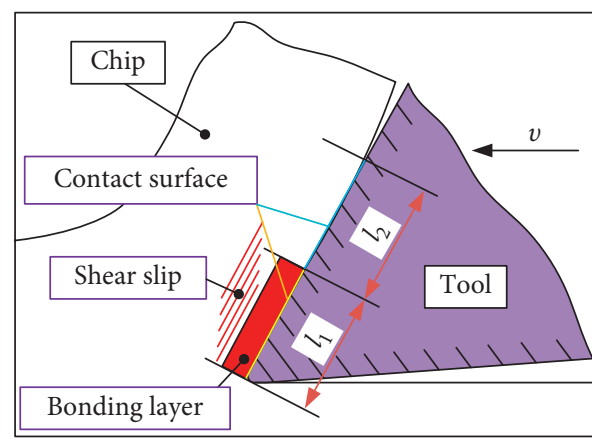

(a)

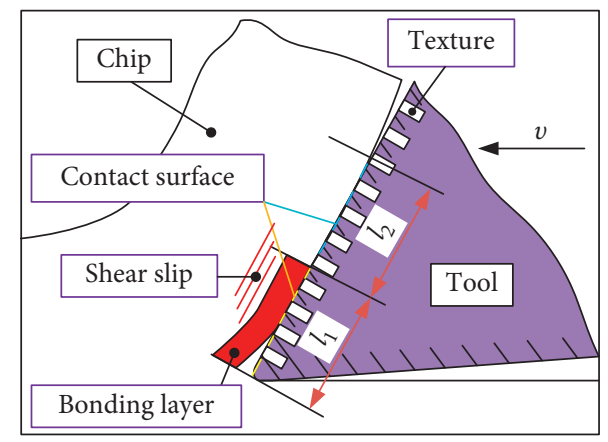

(b)

FIGURE 5: Schematic diagram of bonding between the chip and rake face. (a) Interface bonding of the tool without texture; (b) interface bonding of textured tools.

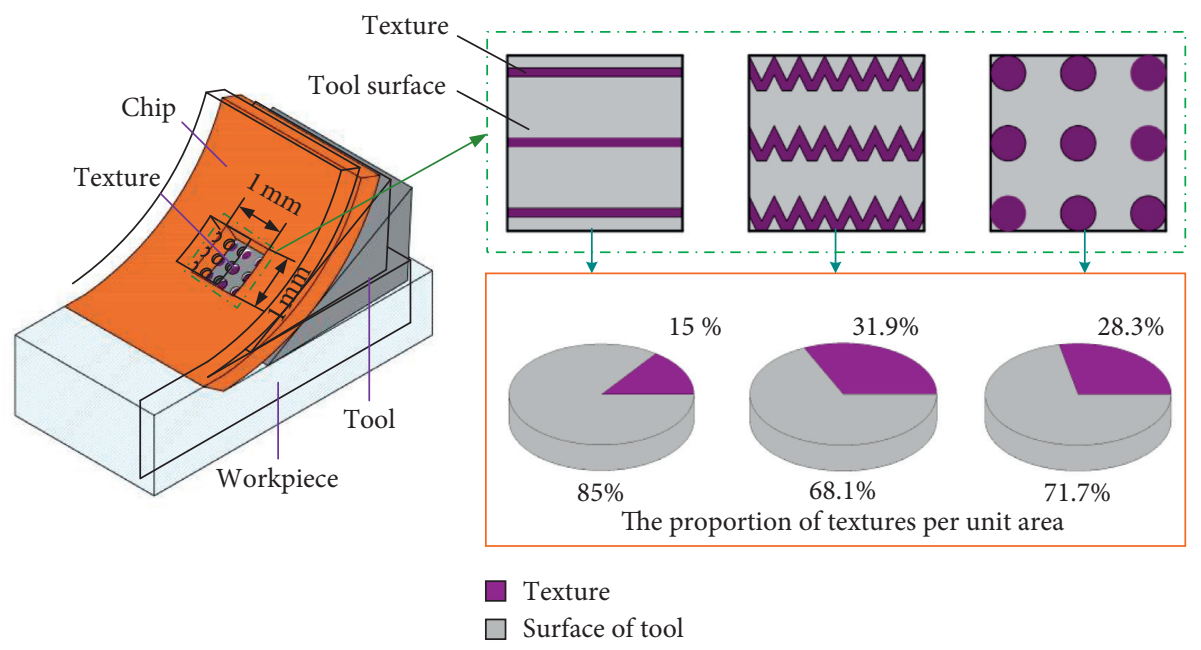

Figure 6: Proportion of the unit area of different textures on the tool surface.

Also, the proportion of burrs on both sides is consistent under different machining conditions. From the statistical chart, it can be concluded that the top-burrs generated by the textured milling tools are significantly reduced. Therefore, it has a beneficial effect on the edge quality of the workpiece when using the textured milling tools. And the wavy texture performs the best, followed by the micropitted texture and the linear texture. Based on the research of Samad Nadimi Bavil and Yigit et al. [29], large forces yielded larger burrs. These observations match the illustration in Section 4.1, which further supports the feasibility of the digital image processing method proposed in this investigation to evaluate top-burrs.

\subsection{Surface Quality}

4.3.1. Surface Morphology. The surface morphology of the processed surface was observed under an ultra-depth of field microscope (type: Keyence VHX-1000C). The left side of Figure 9 shows the surface morphology at a magnification of 500 times, and the right side scans the three-dimensional morphology at 1000 times. It is obvious that the milling textures are more delicate, and the pits are relatively small and less when milling with the wavy textured tool (Figure 9(c)). Similarly, the three-dimensional topography of the protrusions and grooves is thin and shallow. The overall of the surface looks smooth. However, the workpiece has clear furrows, plenty of pits, and large areas of ablation when milling by the nontextured tool, as shown in Figure 9(a). In addition, it can be seen from the three-dimensional topography that the surface has wider protrusions and deeper grooves. The performance of the linear textured tool is better than the micropitted tool, but it is slightly worse than the wavy textured tool (Figures 9(b) and 9(d)). In summary, the surface quality machined by the textured milling tools outperforms that by the nontextured milling tool. Among them, a prominent performance on the surface quality of workpieces can be got by the milling tool with wavy texture.

4.3.2. Roughness $R a$ and $R S m$. The surface roughness is determined by the scratches and the plastic deformation of the material. It is a major indicator of the geometric quality of the workpiece. As the surface roughness decreases, it reflects the better performance of wear resistance, corrosion resistance, and fatigue resistance of the workpiece. In this 


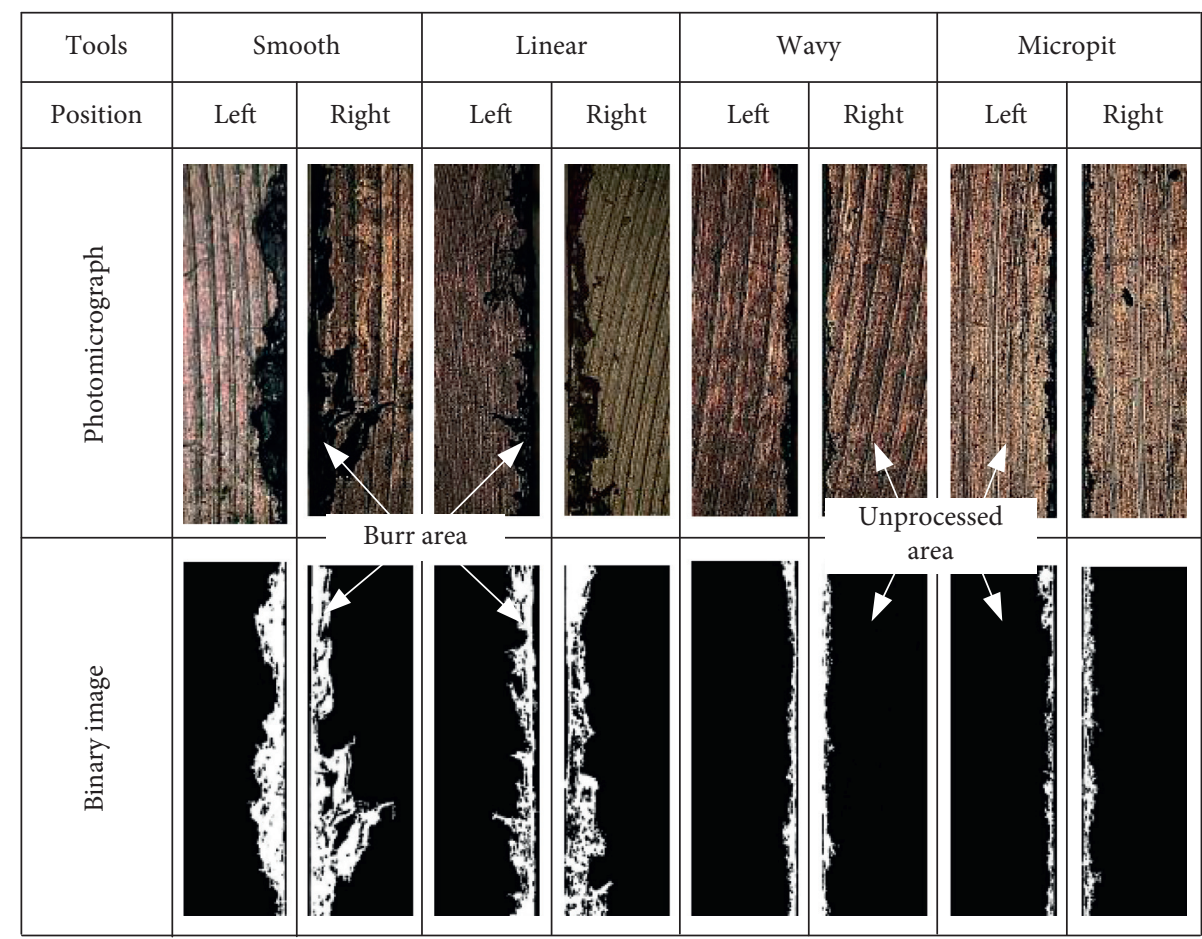

FIgURE 7: Photomicrographs and binary images of the top-burr of the workpiece under different textured milling tools.

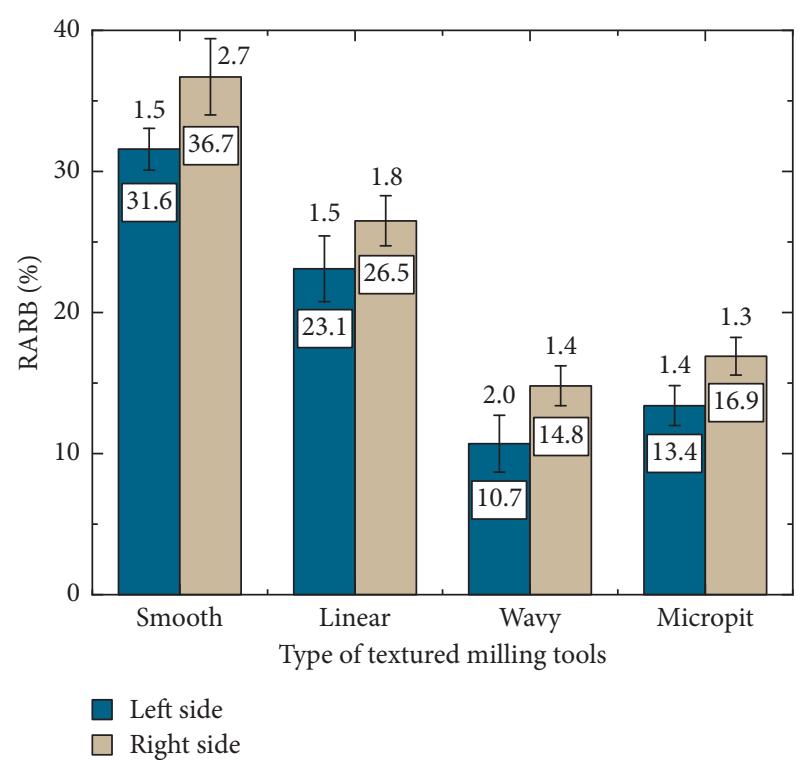

Figure 8: RARB of the workpieces after milling with different textured tools.

experiment, the surface roughness $(\mathrm{Ra})$ was measured by a Mitutoyo surface profiler (type: SJ-210) with a moving speed at $0.5 \mathrm{~mm} / \mathrm{s}$ in accordance with the ISO $13565-1$ standard. Each workpiece is measured five times at the same location, obtaining the average value as shown in Figure 10.

The workpiece machined by the nontextured milling tool has the maximum $\mathrm{Ra}$ of $0.856 \mu \mathrm{m}$ and the RSm of $0.152 \mathrm{~mm}$.
And the minimum $\mathrm{Ra}(0.736 \mu \mathrm{m})$ and $\mathrm{RSm}(0.113 \mathrm{~mm})$ are owned by the wavy textured milling tool. The Ra is $0.773 \mu \mathrm{m}$ when milling by a linear textured milling tool, but it has the maximum RSm of $0.178 \mathrm{~mm}$. Then, the $\mathrm{Ra}$ is $0.845 \mu \mathrm{m}$ and the RSm is $0.124 \mathrm{~mm}$ when milling by the micropitted textured milling tool. From the experimental data, it can be concluded that the $\mathrm{Ra}$ of the workpiece surface after milling by the textured milling tool is less than that by the nontextured milling tool. According to research by Guo et al. [24], the weight-coefficients for Ra reaches $76 \%$ and that for $\mathrm{RSm}$ is $24 \%$, and the roughness of the machined surface was calculated through the weights of $\mathrm{Ra}$ and RSm, thereby evaluating the optimal machined surface. In this experiment, the comprehensive value of the machined surface by a wavy milling tool is 0.438 , and the comprehensive values of a nontextured, linear, and micropitted milling tool are 0.575 , 0.518 , and 0.560 , respectively. In conclusion, the surface quality of the workpiece processed by a textured milling tool is better than that of nontextured milling tool. And the best surface quality can be obtained by preparing the wavy texture on the rake face of a milling tool.

In the process of cutting the metal with a nontextured milling tool, due to the deformation and fracture of the metal, tiny metal debris are randomly generated. These debris, hard metal particles, exist between the blade and chip contact surface. And the soft metal is pushed with the flow of chips, which causes severe furrowing and even glue or bite. Then, it will make the wear more severe between the friction pairs [5], resulting in pits on the surface of the workpiece after processing as shown in Figure 11(a). The texture on the 

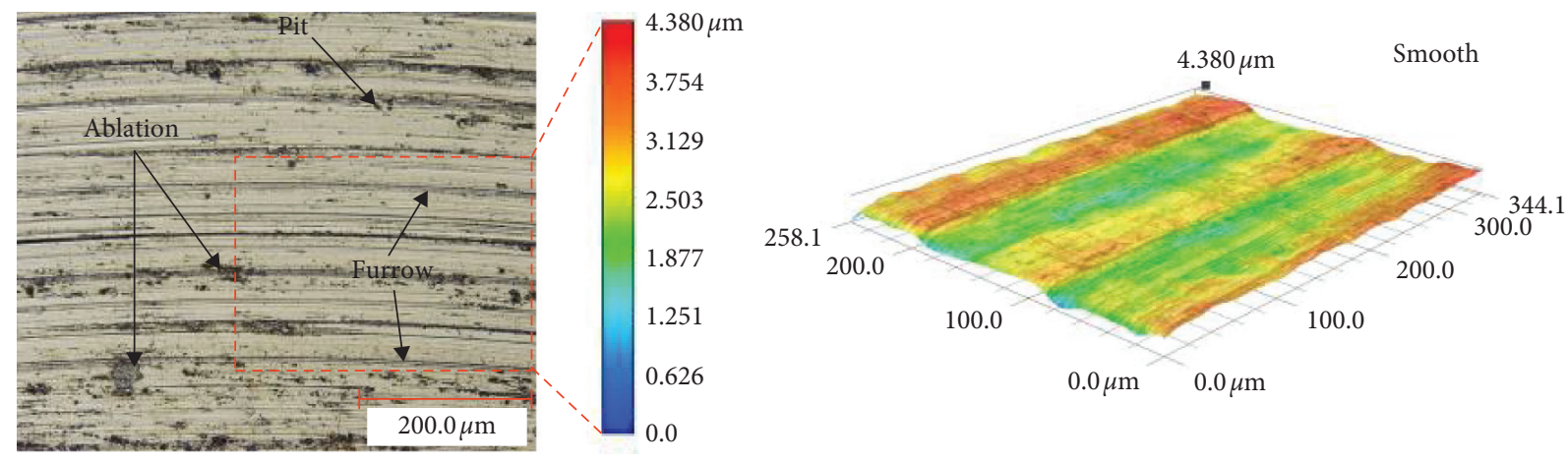

(a)
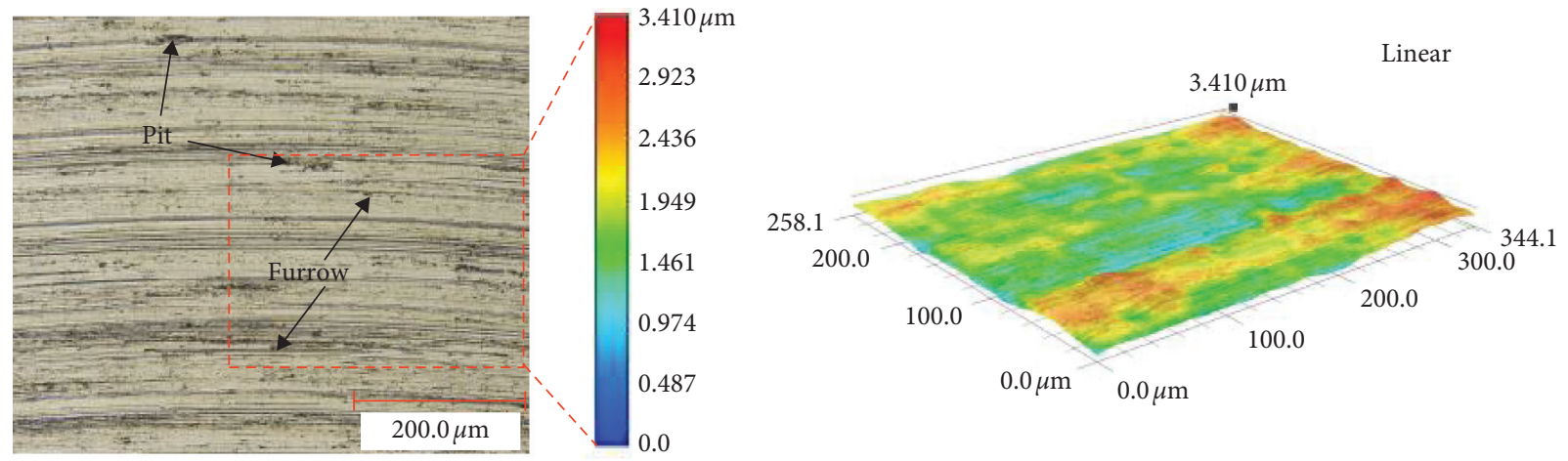

(b)
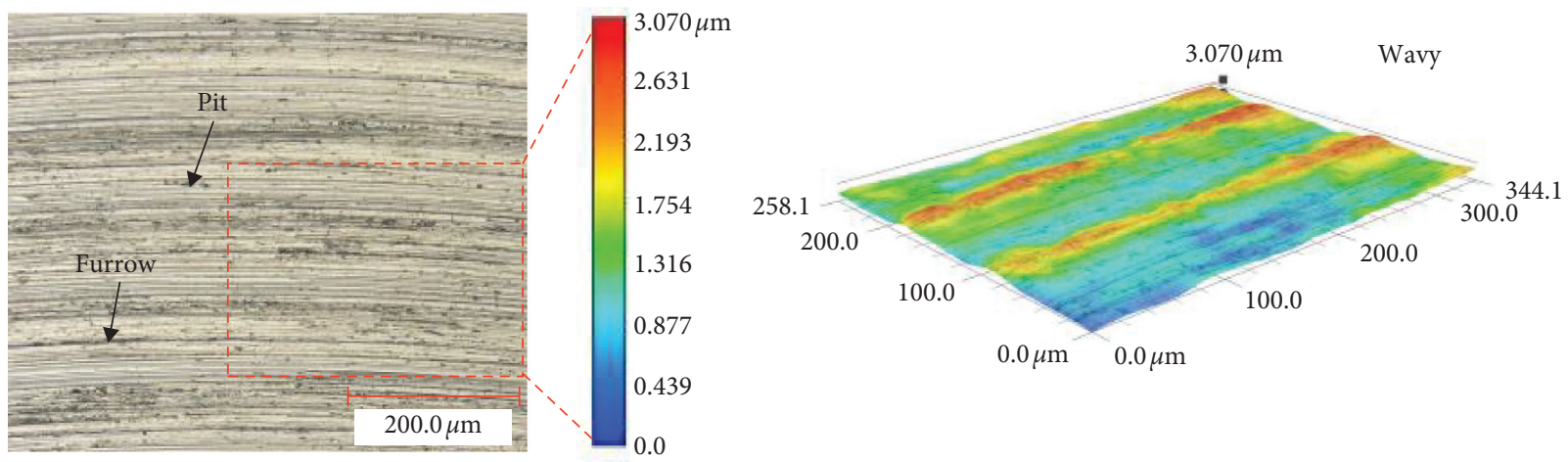

(c)
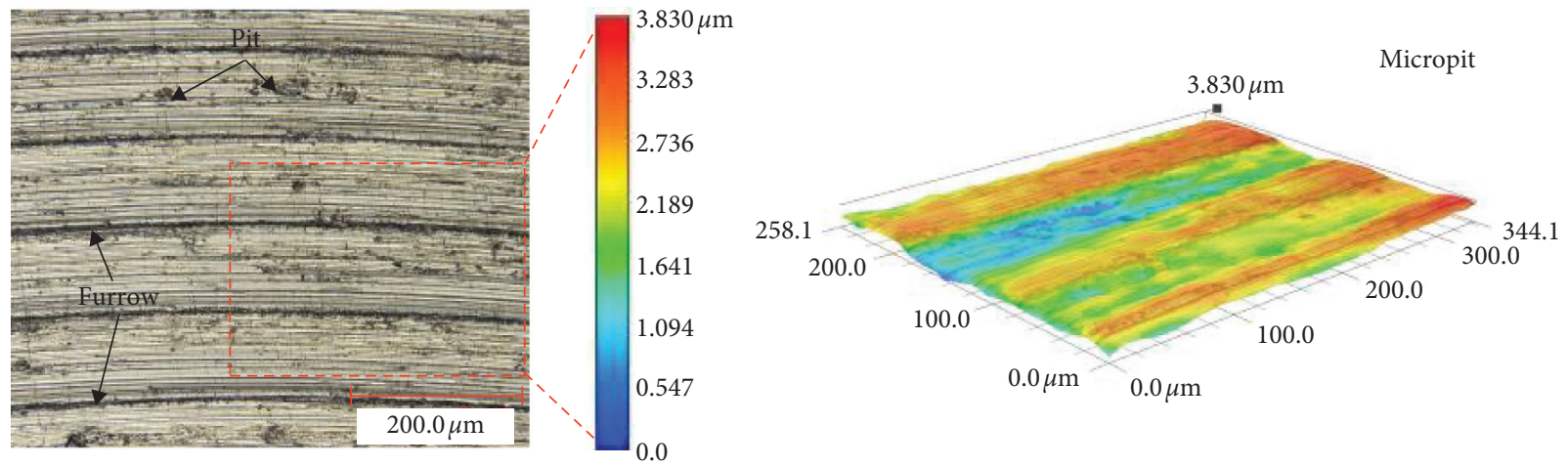

(d)

FIGURE 9: Photomicrographs of workpieces under different textured tool milling. (a) Nontexture, (b) linear texture, (c) wavy texture, and (d) micropit texture. 


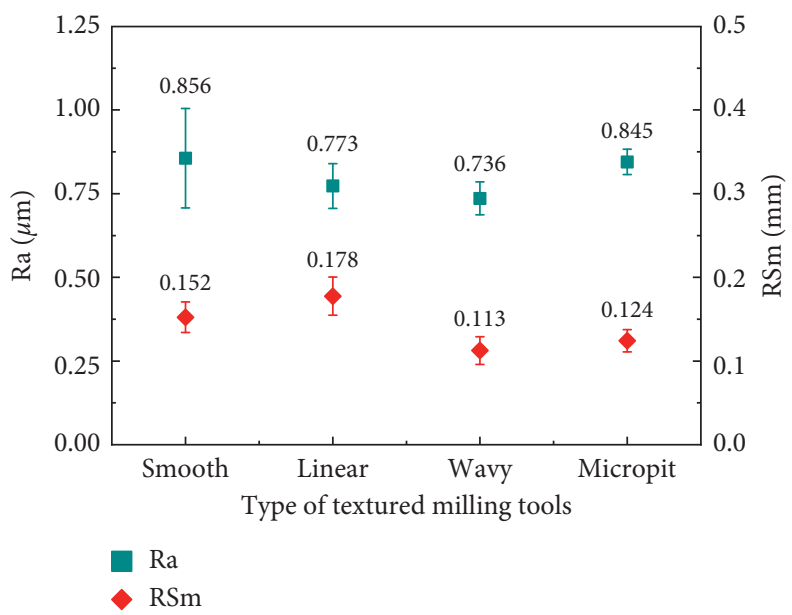

Figure 10: Roughness of the workpiece under different textured tools.

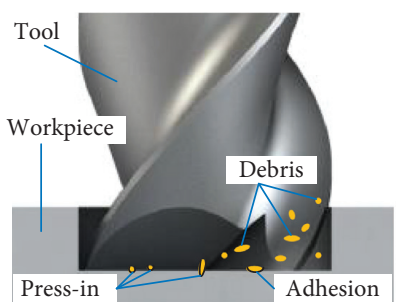

(a)

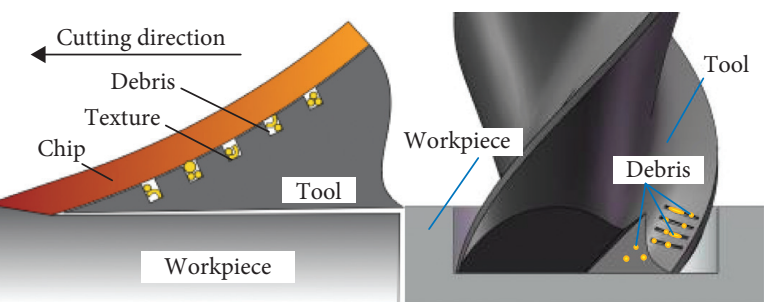

(b)

(c)

FIGURE 11: Schematic diagram of chip retention during milling. (a) Chips fall during milling and damage the machined surface, (b) the principle of texture storage debris, and (c) the use of texture tool reduces the falling of debris during machining.

tool surface plays a role in storing tiny debris by this time. As shown in Figures 11(b) and 11(c), the debris is squeezed into the texture with the relative sliding and friction between the chip and the tool surface during processing. Thus, it reduces the amount of debris falling on the machined surface, while avoiding the surface damage caused by debris which is bonding on the tool or the processed surface. Also, there is almost no large area of ablation on the machined surface of the workpiece. This suggests that textures can reduce debris more effectively by its storage property, thereby improving the machined surface quality of the workpiece. To sum up, the machined surface quality of textured milling tools is better than that of the nontextured milling tool. Outstanding surface quality is obtained due to the optimal storage capacity of a wavy milling tool.

\section{Conclusions}

In this investigation, linear, wavy, and micropitted textures are prepared on the rake face of the end mill. By comparing with nontexture milling tools and textured milling tools, the milling load, top-burr, and surface quality are analyzed. The summary is listed as follows:

(1) Based on data analysis of the cutting load, the textured milling tools have a lower load than the nontexture milling tool, of which the wavy texture is the most significant, and the resultant load Fn is reduced by $10.7 \%$. Due to the existence of texture, the contact area between the tool surface and the chip becomes smaller, so there is less friction, resulting in a lower milling load. The three types of textures are ranked according to the effect of load reduction; the wavy texture is the best, followed by the micropitted textures, and the linear texture has the smallest load reduction.

(2) The image processing technology is used as a means to evaluate the top-burr of the workpiece. The proportion of the burr-pixels is counted by binarizing the photomicrograph, and it is found that the wavy textured milling tool has the least burrs on the top of the workpiece when processing the through groove. In terms of top-burrs, the micropitted texture is slightly larger than the wavy texture, but less than the linear texture. And the textured milling tools play a beneficial role in reducing the top-burr.

(3) The surface quality of the workpiece processed by textured milling tools is better than that milling tool without the texture. Compare the two roughness evaluation indexes $\mathrm{Ra}$ and RSm of the workpiece after milling, and then, comprehensively evaluate the surface roughness through the different weight of $\mathrm{Ra}$ and RSm. The results show that the wavy texture is 
still the best for the surface quality of the workpiece. And the texture has an excellent performance in improving the surface quality because the texture can store debris during processing, and the debris is prevented from being damaged by ploughing and bonding on the processed surface.

\section{Data Availability}

The data used to support the findings of this study are included within the article.

\section{Conflicts of Interest}

The authors declare that there are no conflicts of interest.

\section{Acknowledgments}

This research was supported by the National Natural Science Foundation of China (Grant No. 51775153), China, the National Natural Science Foundation of Zhejiang Province (Grant No. LQ20E050012), China, and the Fundamental Research Funds for the Provincial Universities of Zhejiang (Grant No. GK199900299012-005), China.

\section{References}

[1] H. Wang, J. Yang, and F. Sun, "Cutting performances of MCD, SMCD, NCD and MCD/NCD coated tools in highspeed milling of hot bending graphite molds," Journal of Materials Processing Technology, vol. 276, Article ID 116401, 2020.

[2] Y. Li, J. Deng, Y. Chai, and W. Fan, "Surface textures on cemented carbide cutting tools by micro EDM assisted with high-frequency vibration," The International Journal of Advanced Manufacturing Technology, vol. 82, no. 9-12, pp. 2157-2165, 2016.

[3] G. Byrne, D. Dornfeld, and B. Denkena, "Advancing cutting technology," CIRP Annals, vol. 52, no. 2, pp. 483-507, 2003.

[4] S. Tatsuya and E. Toshiyuki, "Improving anti-adhesion in aluminum alloy cutting by micro stripe texture," Precision Engineering, vol. 36, no. 2, pp. 229-237, 2012.

[5] S. Niketh and G. L. Samuel, "Surface texturing for tribology enhancement and its application on drill tool for the sustainable machining of titanium alloy," Journal of Cleaner Production, vol. 167, pp. 253-270, 2017.

[6] W. Reiner, S. Johannes, and G. Peter, "In situ observation of cavitation in crossed microchannels," Tribology International, vol. 55, pp. 81-86, 2012.

[7] G. K.-H. Zum, R. Wahl, and K. Wauthier, "Experimental study of the effect of micro-texturing on oil lubricated ceramic/steel friction pairs," Wear, vol. 267, no. 5-8, pp. 1241-1251, 2009.

[8] H. L. Costa and I. M. Hutchings, "Effects of die surface patterning on lubrication in strip drawing," Journal of $\mathrm{Ma}$ terials Processing Technology, vol. 209, no. 3, pp. 1175-1180, 2009.

[9] W. Reiner, S. Johannes, and G. Peter, "Influence of the real geometry of the protrusions in micro textured surfaces on frictional behaviour," Tribology Letters, vol. 47, no. 3, pp. 447-453, 2012.

[10] S. Tatsuya and E. Toshiyuki, "Development of a cutting tool with a nano/micro-textured surface-improvement of anti- adhesive effect by considering the texture patterns," Precision Engineering, vol. 33, no. 4, pp. 425-429, 2009.

[11] P. Koshy and J. Tovey, "Performance of electrical discharge textured cutting tools," CIRP Annals, vol. 60, no. 1, pp. 153-156, 2011.

[12] O. Toshiyuki, K. Akihiro, T. Hidemitsu, and O. Akira, "Microtexture at the coated tool face for high performance cutting," International Journal of Machine Tools and Manufacture, vol. 51, no. 12, pp. 966-972, 2011.

[13] L. Tiffany Davis, P. Z. Liu, S. W. Xiong et al., "Surface texturing of drill bits for adhesion reduction and tool life enhancement," Tribology Letters, vol. 52, no. 1, pp. 113-122, 2013.

[14] J. Xie, M. J. Luo, K. K. Wu, L. F. Yang, and D. H. Li, "Experimental study on cutting temperature and cutting force in dry turning of titanium alloy using a non-coated microgrooved tool," International Journal of Machine Tools and Manufacture, vol. 73, pp. 25-36, 2015.

[15] K. Noritaka, O. Kazuma, M. Noboru, N. Kazuhito, and Y. Makoto, "Development and machining performance of a textured diamond cutting tool fabricated with a focused ion beam and heat treatment," Precision Engineering, vol. 47, pp. 311-320, 2017.

[16] A. Naveed, D. Saied, and A. M. Abdulrehman, "Laser ablation and laser-hybrid ablation processes: a review," Materials and Manufacturing Processes, vol. 31, no. 9, pp. 1121-1142, 2016.

[17] F. Anis, J. W. David, and T. M. Paul, "Femtosecond laser surface structuring of carbide tooling for modifying contact phenomena," Proceedings of the Institution of Mechanical Engineers, Part B: Journal of Engineering Manufacture, vol. 228, pp. 1325-1337, 2014.

[18] Y. Liu, J. Deng, F. Wu, R. Duan, X. Zhang, and Y. Hou, "Wear resistance of carbide tools with textured flank-face in dry cutting of green alumina ceramics," Wear, vol. 372-373, pp. 91-103, 2017.

[19] D. Vasumathy and A. Meena, "Influence of micro scale textured tools on tribological properties at tool-chip interface in turning AISI 316 austenitic stainless steel," Wear, vol. 376377, pp. 1747-1758, 2017.

[20] S. Manel and S. Kumar, "Heat generation and temperature in orthogonal machining," International Journal of Scientific \& Engineering Research, vol. 8, no. 2, pp. 31-33, 2017.

[21] L. K. Gillespie and P. T. Blotter, "The formation and properties of machining burrs," Journal of Engineering for Industry, vol. 98, no. 1, pp. 66-74, 1976.

[22] J. Blotter and H. Tritschler, "Micro cutting of steel," Microsystem Technologies, vol. 10, no. 3, pp. 167-174, 2004.

[23] M. Chen, G. Liu, and Z. Shen, "Study on active process control of burr formation in $\mathrm{Al}$-alloy milling process," in Proceedings of the IEEE, International Conference on Automation Science and Engineering, pp. 431-436, Shanghai, China, October 2006.

[24] S. Guo, C. Li, Y. Zhang et al., "Experimental evaluation of the lubrication performance of mixtures of castor oil with other vegetable oils in MQL grinding of nickel-based alloy," Journal of Cleaner Production, vol. 140, pp. 1060-1076, 2017.

[25] K. Johannes, G. Jens, M. Erich, S. Reinhard, S. Volker, and W. Alexander, "Detailed analysis of microstructure of intentionally formed built-up edges for improving wear behaviour in dry metal cutting process of steel," Wear, vol. 311, no. 1-2, pp. 21-30, 2014.

[26] P. Yan, Y. Rong, and G. Wang, "The effect of cutting fluids applied in metal cutting process," Proceedings of the Institution of Mechanical Engineers, Part B: Journal of Engineering Manufacture, vol. 230, no. 1, pp. 19-37, 2016. 
[27] N. Seyed Ali and S. Victor, "Factors governing burr formation during high-speed slot milling of wrought aluminium alloys," Proceedings of the Institution of Mechanical Engineers, Part B: Journal of Engineering Manufacture, vol. 227, no. 8, pp. 1165-1179, 2013.

[28] N. Seyed Ali and S. Victor, "Milling burr formation, modeling and control: a review," Proceedings of the Institution of $\mathrm{Me}-$ chanical Engineers, Part B: Journal of Engineering Manufacture, vol. 229, no. 6, pp. 893-909, 2015.

[29] O. Samad Nadimi Bavil and K. Yigit, "Built-up edge effects on process outputs of titanium alloy micro milling," Precision Engineering, vol. 49, pp. 305-315, 2017. 